\title{
Substation intelligent condition acquisition controller based on IEC61850 for online-monitoring IED
}

\author{
Juan Liu and Guang Chen \\ State Grid Electric Power Research Institute, \\ Beijing, 102200, China \\ 'E-mail: liujuan6@sgepri.sgcc.com.cn
}

\begin{abstract}
This paper discusses the condition acquisition controller's realization method based on IEC 61850 standard communication protocol. It presents a design scheme about main structure and key technologies. First, it studied the device's hardware requirements, communication environment, work flow, then designed the device's communication service model and function based on IEC61850 with CMU, provided the interaction logic and the communication process with CAG, the Web access function. Finally, this paper also introduces the method of its implementation. The device is successfully used in smart substation, lay a good foundation for the realization of smart grid's information, automation and interaction.
\end{abstract}

Keywords: Transformation Equipment; Condition; Controller; Monitoring; Intelligent.

\section{Introduction}

The on-line monitoring device of the conventional substation is related to different professionals, and the factory is more. In general, the station has multiple sets of monitoring system, it is difficult to share information and can't carry out a comprehensive analysis based on various types of monitoring data on the operational status of the equipment .

In order to realize the unified maintenance and comprehensive utilization and diagnosis analysis of monitoring data, the intelligent condition acquisition controller(CAC) is introduced into the substation condition monitoring system [1-3]. In the substation, CAC is the key device of state monitoring system of intelligent substation [4], it connecting main station of equipment condition monitoring system and IEDs. The sensors which have access to it included the transformer oil chromatogram, transformer core grounding, SF6 gas monitoring, and switching characteristics monitoring, GIS partial discharge monitoring, capacitive equipment insulation monitoring, MOA insulation monitoring, storage battery online monitoring and other series. The device can bring convenience to the maintenance of substation[5], it uses the IEC 61850 standard 
unified monitoring device communication protocol, unified monitoring data format and unified interface, realized the IED's plug and play and interoperability. The device is helpful to clear understanding of the development trend of running state, find potential problems of the key device.

\subsection{Overall design of CAC}

\subsubsection{System structure}

Figure 1 shows the hierarchical and distributed network structure of the condition monitoring system, it is composed of process layer, bay layer and station layer. CAC is installed in the station control layer, monitoring the substation, achieved the operation control, storage and forwarding of state data of the whole state monitoring system .

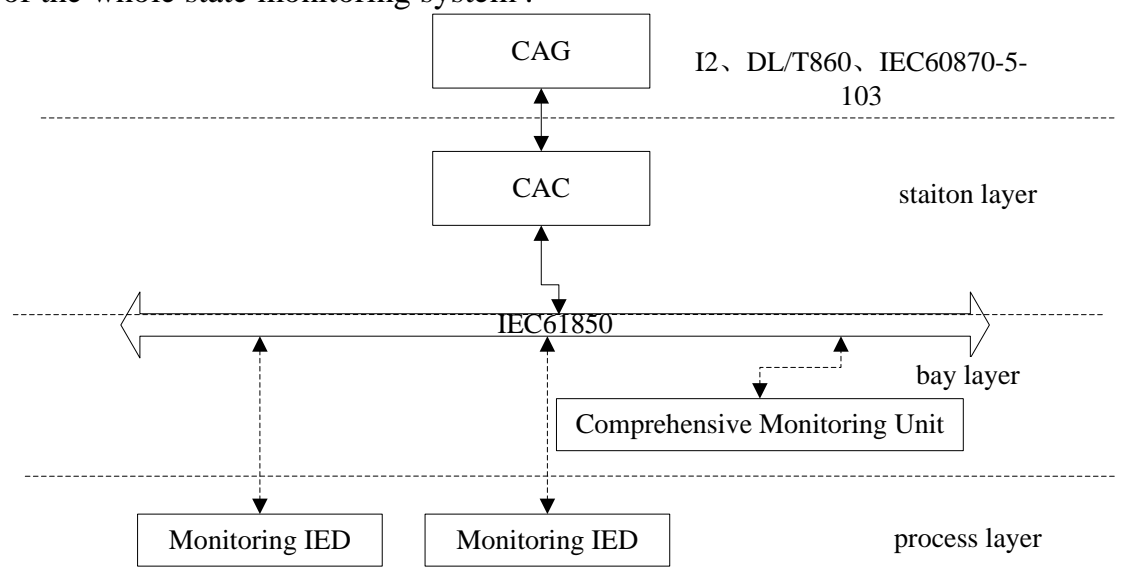

Fig. 1. The architecture of Smart Grid Power equipment condition monitoring system

Among them, CAG(Condition acquisition gateway) deployed in the master station system, receiving the master station system data of CAC. CMU (Comprehensive Monitoring Unit) is a comprehensive monitoring unit, deployed in the bay layer, receiving and processing the sending state monitoring device data, CAC implementation and standardization of data communication.

\subsubsection{The System consists}

CAC includes three parts: configuration management tools, communication unit and the Web platform. configuration management tool is for the configuration and management of the whole station online monitoring. The communication unit software takes the whole substation as the object, manages the all monitoring communication data ,all IEDs and CMUs, implements the comprehensive analysis of the monitoring data, warning function, and the 
control functions to IED and CMU's which have parameter setting, data calls, synchronize time, forced to restart and the others, and can carry on the standardized communication with the main station. Web platform provides visible display and query function of monitoring data. The system function structure diagram as shown in Figure 2.

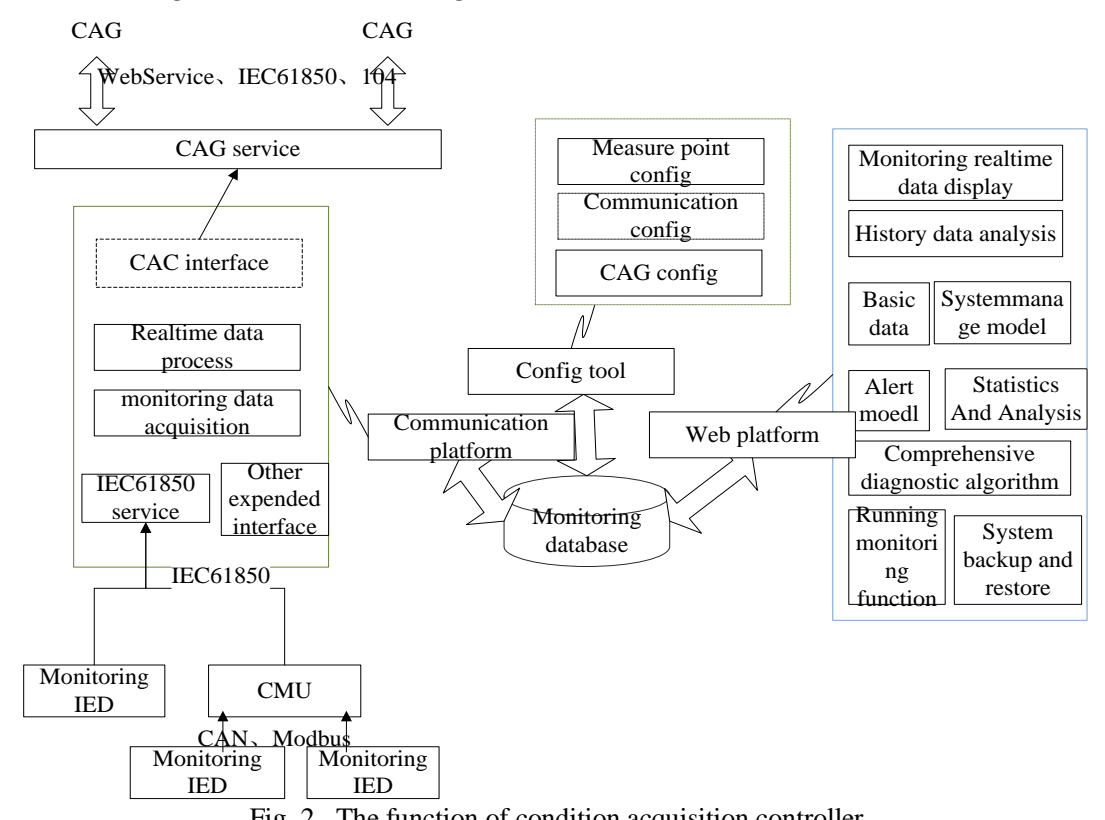

Fig. 2. The function of condition acquisition controller.

\subsubsection{Hardware requirements}

CAC designed with high performance embedded industrial control computer without fan as the foundation, using standard rack mounting structure, equipped with optical ethernet switch; has multiple ethernet port, a plurality of optical Ethernet isolation serial, wide input voltage, wide operating temperature.

\subsubsection{Communication environment}

- $\quad$ optical fiber communication port: between station layer and bay layer of stations, optical fiber transmission rate is100Mbps.

- the Ethernet communication port: between control layer and master station or other system in station. 


\section{The Key Technologies of CAC}

\subsection{The work flow}

First, system startup with configuration and management of the whole station online monitoring by the substation configuration tool, then get IED running state, sent collection of monitoring data and other IED information to CAG in each CMU, then provides the user to view the substation information through the Web platform.

\subsection{Working principle}

Between CAC and the CMU communication with IEC61850 communication protocol and client / server communication model. CAC provide the function of client, CMU as a server, they realize the communication connection, alarm, summon, periodic upload, setting value and control, model, data query access communication function, as shown in Figure 3.

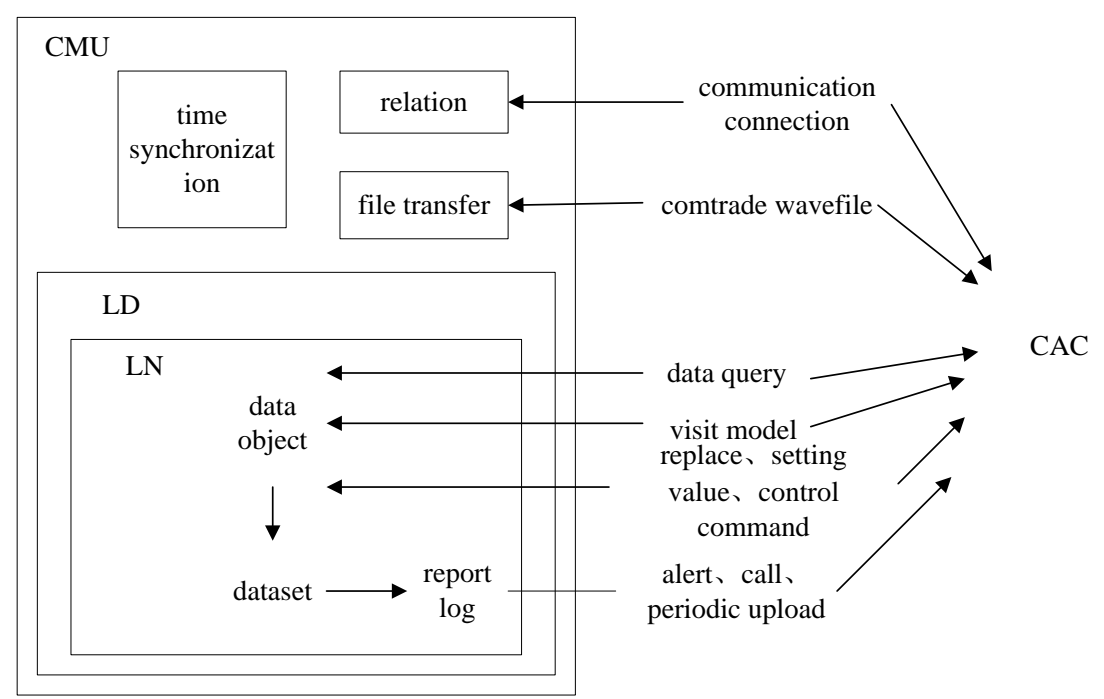

Fig. 3 Communication between CAC and CMU

\subsection{Interactive logic of CAC and CAG}

$\mathrm{CAC}$ and $\mathrm{CAG}$ access to each other's published communication interface by means of Web service. The services were included in Table 1. 
Table 1. The Web services of CAC and CAG

\begin{tabular}{lll}
\hline CAC & CAG & \\
\hline \multirow{3}{*}{ DownloadCAGCtrl } & Upload Heartbeat Info & Upload monitor data \\
& Upload CACC on fig & Get Newest Version \\
& Get Update File & Get History Version \\
\hline
\end{tabular}

In Figure 4, CAC timing on data send cycle time which has been set as fix value, timing begins with receive heartbeat signal and time synchronize complete. CAG can send GETCONFIG, SETCONFIG, RESEND, GETDATA commands according to need.

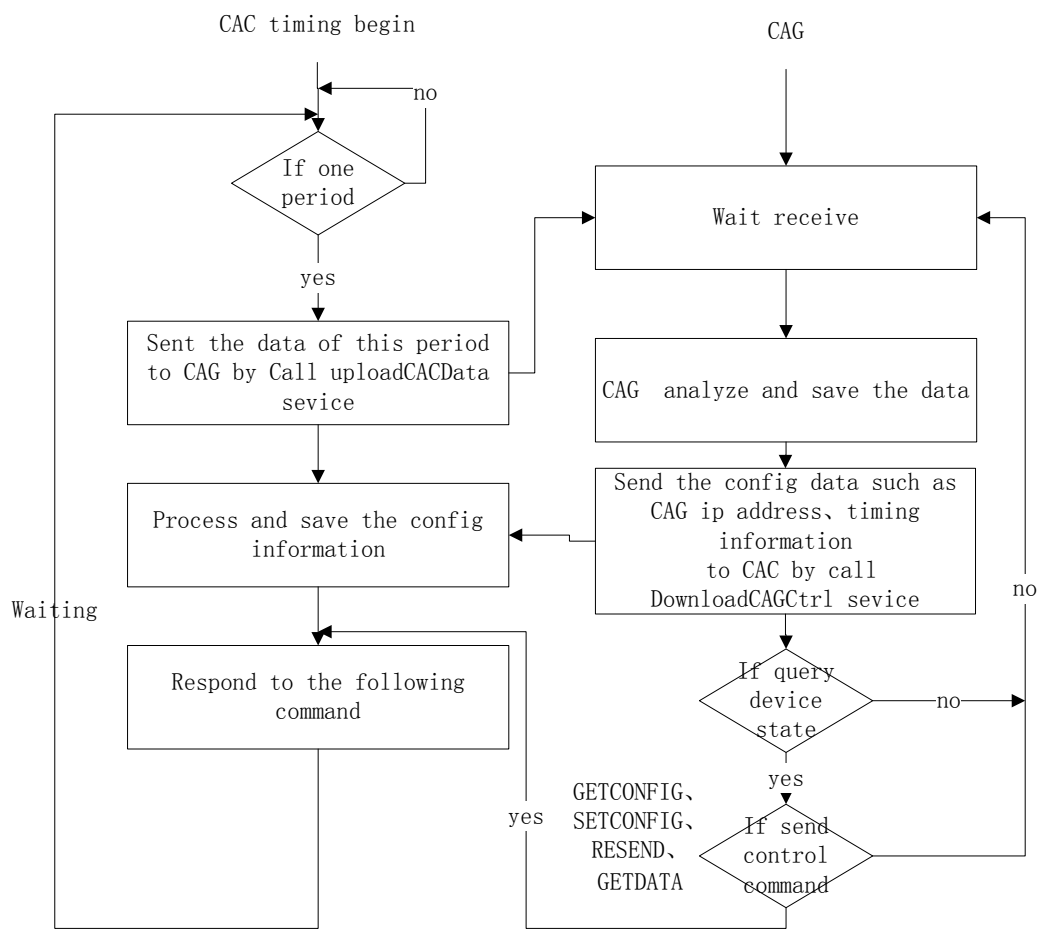

Fig. 4. The interaction process of $\mathrm{CAC}$ and $\mathrm{CAG}$

\subsection{The function of Web platform}

CAC device's web display function includes view online data monitoring, analysis historical data, statistical analysis, comprehensive diagnosis algorithm, real-time condition monitoring etc. 


\section{Implementation and Application}

CAC's communication unit software developed using VC++, its Web platform developed using C\# and MySQL database, The system supports CAN, RS232/485, Ethernet interface.

At present this kind of device has been successful application of intelligent substation in Shanghai, Zhejiang and other places in China.

\section{Summary}

CAC is an effective solution to multi type, multi manufacturer, functions repetition, data cannot be shared and other issues of the current substation monitoring system. It is give a unified information model and communication protocol and is conducive to station data resources integration, to equipment information standardization. It provides data support for the equipment fault diagnosis, has good application prospects.

\section{References}

1. National power system management and information exchange Standardization Technical Committee, DL/T 860 communication networks and systems in substations( China Electric Power Press ,Beijing,2006).

2. China State Grid Corp, Q/GDW 739 - 2012 power transmission and transformation equipment online monitoring system of substation equipment online monitoring interface of II network communication specification (China Electric Power Press ,Beijing,2012).

3. China State Grid Corp., Q/GDW 740 - 2012 power transmission and transformation equipment online monitoring system of substation equipment online monitoring interface of 12 network communication specification (China Electric Power Press , Beijing,2012).

4. DL/T $1146-2009$ DL/T 860 implementation of technical specification. Beijing: Technical Committee for standardization management and the exchange of information on national power system(2009)

5. Xiao-Feng Li, Juan Liu, Shao-Xian Qin, Key technology for power transmission and transfor-mation equipment's online monitoring system based on IEC61850, High Voltage Engineering, 36 (12) 3041-3046(2010). 